\title{
Imaginary Cubic Perturbation: Numerical and Analytic Study
}

\author{
Jean Zinn-Justin, 1 * and Ulrich D. Jentschura ${ }^{2}$ \\ ${ }^{1}$ CEA/IRFU, Centre de Saclay, 91191 Gif-sur-Yvette Cedex, France \\ ${ }^{2}$ Missouri University of Science and Technology, Rolla, Missouri 65409-0640, USA
}

\begin{abstract}
The analytic properties of the ground state resonance energy $E(g)$ of the cubic potential are investigated as a function of the complex coupling parameter $g$. We explicitly show that it is possible to analytically continue $E(g)$ by means of a resummed strong coupling expansion, to the second sheet of the Riemann surface, and we observe a merging of resonance and antiresonance eigenvalues at a critical point along the line $\arg (g)=5 \pi / 4$. In addition, we investigate the convergence of the resummed weak-coupling expansion in the strong coupling regime, by means of various modifications of order-dependent mappings (ODM), that take special properties of the cubic potential into account. The various ODM are adapted to different regimes of the coupling constant. We also determine a large number of terms of the strong coupling expansion by resumming the weak-coupling expansion using the ODM, demonstrating the interpolation between the two regimes made possible by this summation method.
\end{abstract}

PACS numbers: 11.10.Jj, 11.15.Bt, 11.25.Db, 12.38.Cy, 03.65.Db

\section{INTRODUCTION}

The Hamiltonian

$$
H=-\frac{1}{2} \frac{\partial^{2}}{\partial x^{2}}+\frac{1}{2} x^{2}+\mathrm{i} \frac{\sqrt{g}}{6} x^{3},
$$

with $g$ real positive, has been a subject of a number of studies since it has been conjectured by Bessis and ZinnJustin (1992) to have a real spectrum. Arguably, Eq. (1) provides the simplest example of a $\mathcal{P} \mathcal{T}$ symmetric Hamiltonian, that is, of a Hamiltonian invariant under a simultaneous complex conjugation (i.e., time reversal) and parity transformation $x \mapsto-x$ (see Ref. [1]). In particular, the reality of its spectrum was proven first in the strong coupling limit [2], then more generally in Ref. 3] (together with the positivity of the eigenvalues).

Here, we continue our investigations [4] regarding its numerical and analytic properties, including the analytic structure of its resonance energy eigenvalues as a function of the complex coupling parameter $g$, specially in the strong coupling regime and near the first level crossing singularity.

We denote by $E(g)$ an energy eigenvalue of the Hamiltonian (11). One verifies that its perturbative expansion contains only integer powers of $g$ :

$$
E(g) \underset{g \rightarrow 0}{\sim} \sum_{L=0} E_{L} g^{L},
$$

with real coefficients $E_{L}$. The series is divergent for all $g$, but is Borel summable [5, [6], and a steepest descent calculation of the path integral representation of the corresponding quantum partition function [7-10] yields a large order behaviour for the $n$th energy eigenvalue of the form

$$
E_{L} \underset{L \rightarrow \infty}{\sim}(-1)^{L+1} \frac{6}{\pi^{3 / 2}} \frac{288^{n}}{n !} \frac{\Gamma\left(L+n+\frac{1}{2}\right)}{A^{L+n+1 / 2}},
$$

*Electronic address: jean.zinn-justin@cea.fr where $A=\frac{24}{5}$ is the instanton action.

More recently, Padé summability was also rigorously established in Ref. 11]. The imaginary part of the energy levels on the cut for $g=-|g|+\mathrm{i} 0$ is positive. This proof confirms numerical investigations based on the summation of the perturbative expansion by Padé approximants [12].

More information on analytic and numerical properties was provided by an ODM summation [13, 14] of the perturbative series for the ground state energy (see Ref. [4]). It was based on the mapping $g \mapsto \lambda$,

$$
g=\rho \frac{\lambda}{(1-\lambda)^{5 / 2}},
$$

where $\rho$ is an order-dependent parameter.

In particular, the leading term of the strong coupling expansion could be determined (and was found in agreement with results coming direct solutions of Schrödinger equation [15, 16]) and the positivity of the imaginary part verified numerically for all $g<0$. It was shown that along the negative real axis, the imaginary part above the cut is a simple positive decreasing function interpolating smoothly between a strong coupling power-law behaviour and a non-analytic exponential tunneling factor obtained from semi-classical instanton calculation for weak coupling. Finally, it was argued that the ground state energy is analytic up to $\arg (g)=5 \pi / 4$ in the second Riemann sheet, a property that we discuss in this article in more detail.

By a simple coordinate translation, one can relate the Hamiltonian (1) to another $\mathcal{P} \mathcal{T}$ symmetric Hamiltonian,

$$
H^{[\mathrm{qc}]}=-\frac{1}{2} \frac{\partial^{2}}{\partial x^{2}}+\mathrm{i}\left(\frac{1}{6} x^{3}+\frac{1}{2} \chi x\right),
$$

where $\chi=g^{-4 / 5}$. This correspondence suggests to sum the series by another ODM of the form

$$
g=\rho \frac{\lambda}{(1-\lambda)^{5 / 4}}
$$


which is shown here to be much more efficient in the strong coupling regime, while the mapping (4) (and a variation thereof discussed below) is more efficient for weak coupling. The method (6) is used here in order to determine a number of coefficients of the large $g$ expansion (small $\chi$ expansion). Finally, studying the limit of weak $\chi$, we also construct a corresponding continued fraction (a subclass of Padé approximants), which enables us to obtain more insight into the analytic structure.

Combining all methods, we have a precise control on the analytic continuation of the ground state energy in the first Riemann sheet of the uniformization variable $\chi=g^{-4 / 5}$, except near the cut in the $\chi$-plane. Based on our observation, we conjecture that the ground state energy of the Hamiltonian (5) is a real analytic function, with a cut on the real negative $\chi$ axis, which corresponds to a complex phase of $\arg (g)=5 \pi / 4$ for the original coupling parameter $g$ and thus lies on the second sheet of the Riemann surface for the original coupling parameter $g$. This domain is not covered by Ref. [3]. The imaginary part of the ground state energy on the cut for $\chi=-|\chi|+$ $\mathrm{i} 0$ is positive. The small $\chi$ expansion of the resonance energies converges in a disk. From the point of view of the initial Hamiltonian (11), the singularity closest to the origin has the interpretation of a level crossing.

This can be seen as follows: the cut for negative $\chi$ corresponds to a cut of the energy level on the second sheet [17-19] of the Riemann surface ("Bender-Wu cut"). It starts from the limit of the circle of convergence of the strong coupling expansion, i.e., from a point on the boundary of the circle of convergence of the $\chi$ expansion. Two resonance energies are attached to points infinitesimally displaced above and below the cut. The functions describing the real parts of the two resonance energies are equal on the cut. They cross as we cross the cut. By contrast, the functions describing their imaginary parts are equal and opposite on the cut and vary smoothly in its immediate vicinity.

At the start of the cut, the imaginary part of the resonance and antiresonance energy is zero, but the real parts still cross, and this configuration therefore corresponds to a level crossing, given by the confluence of two energy levels on the second sheet of the Riemann surface characterizing the resonance energies as a function of $g$. For the Hamiltonian (5), it corresponds, in addition, to a spontaneous breaking of the $\mathcal{P} \mathcal{T}$ symmetry (namely, the spectrum composed of the resonance and antiresonance energies with their equal and opposite imaginary parts corresponds to a manifestly non- $\mathcal{P} \mathcal{T}$ symmetric time evolution). Starting from the region where the two eigenvalues merge and are real, we can say that after the singularity, they become complex conjugate.

To confirm this picture, that is, analyticity in a cutplane in the $\chi$ variable, sign of the imaginary part of the ground state energy on the cut and level merging singularity we investigate together the ground state and the first excited state, forming symmetric combinations: sum and difference squared of the corresponding energies. We verify that these functions are regular at the level merging singularity, showing that the level merging involves the ground state and the first excited state. This gives us a totally consistent picture and, moreover, allows us to determine with increased precision a number of terms of the small $\chi$ expansion. In turn, from the small $\chi$ expansion a continued fraction expansion is derived, whose coefficients are all positive and seem to converge to a value consistent with a square root singularity, within errors.

As a final remark, we notice that the Hamiltonian (1) can be considered as a toy model for the $\mathrm{i} \phi^{3}$ quantum field theory in two and three space dimensions, whose renormalization group functions describe the Lee-Yang edge singularity of Ising-like statistical models 20, 21].

This paper is organized as follows. We first discuss the scaling of the coupling constant and the related strong coupling Hamiltonians in Sec. III. General properties of ODM summation methods are recalled in Sec. III. Optimized methods for the problem at hand are introduced in Sec. IV] Numerical results for $g$ positive and negative are obtained and discussed in Sec. V] Sec. VI is devoted to the strong coupling expansion and level merging. Analytic properties of the ground state resonance in the second Riemann sheet and continued fraction expansion are discussed in Sec. VII Conclusions are relegated to Sec. VIII.

\section{STRONG COUPLING HAMILTONIANS}

The time-independent Schrödinger equation

$$
\left(-\frac{1}{2} \frac{\partial^{2}}{\partial x^{2}}+\frac{1}{2} x^{2}+\frac{\mathrm{i}}{6} \sqrt{g} x^{3}\right) \psi(x)=E(g) \psi(x)
$$

corresponds to the Hamiltonian (1). With proper boundary conditions at infinity, this equation determines the eigenvalues $E(g)$ of the operator.

After rescaling the variable $x$ as $x \mapsto g^{-1 / 5} x$, the eigenvalue equation can be rewritten as follows (the superscript [sc] stands for the strong-coupling limit)

$$
H^{[\mathrm{sc}]}(\xi) \psi(x)=E^{[\mathrm{sc}]}(\xi) \psi(x),
$$

where

$$
\xi=g^{-2 / 5},
$$

and the strong coupling Hamiltonian is

$$
H^{[\mathrm{sc}]}(\xi)=-\frac{1}{2} \frac{\partial^{2}}{\partial x^{2}}+\frac{\mathrm{i}}{6} x^{3}+\frac{1}{2} \xi x^{2} .
$$

The energies of the original and the strong coupling Hamiltonian are related by

$$
E(g)=g^{1 / 5} E^{[\mathrm{sc}]}\left(g^{-2 / 5}\right)=g^{1 / 5} E^{[\mathrm{sc}]}(\xi) .
$$


In particular, we have in leading order

$$
E(g) \underset{g \rightarrow \infty}{\sim} g^{1 / 5} E^{[\mathrm{sc}]}(0),
$$

and the strong coupling expansion

$$
E^{[\mathrm{scc}]}(\xi)=\sum_{L=0}^{\infty} E_{L}^{[\mathrm{sc}]} \xi^{L} .
$$

In the uniformizing variable $\sqrt{\xi}=g^{-1 / 5}$, the singularities of $E(g)$ in the complex plane correspond to level crossings, whereas the cut of the original resonance energy, defined as a function of $g$, is moved to $\xi=\infty$.

We then shift the coordinate $x \mapsto x+\mathrm{i} \xi$. The equation becomes

$$
\left(-\frac{1}{2} \frac{\partial^{2}}{\partial x^{2}}+\frac{\mathrm{i}}{6} x^{3}+\frac{\mathrm{i}}{2} \xi^{2} x-\frac{1}{3} \xi^{3}\right) \psi(x)=E^{[\mathrm{sc}]}(\xi) \psi(x) .
$$

We conclude that

$$
E^{[\mathrm{sc}]}(\xi)=-\frac{1}{3} \xi^{3}+E^{[\mathrm{qc}]}\left(\xi^{2}\right),
$$

where $E^{[q c]}\left(\chi \equiv \xi^{2}\right)$ is a function of the linear coupling

$$
\chi=\xi^{2}=g^{-4 / 5},
$$

and thus an eigenvalue of the $\mathcal{P} \mathcal{T}$ symmetric Hamiltonian (5) corresponding to the Schrödinger equation

$$
H^{[\mathrm{qc}]}(\chi) \psi(x)=E^{[\mathrm{qc}]}(\chi) \psi(x),
$$

where

$$
H^{[\mathrm{qc}]}(\chi)=-\frac{1}{2} \frac{\partial^{2}}{\partial x^{2}}+\mathrm{i}\left(\frac{1}{6} x^{3}+\frac{1}{2} \chi x\right) .
$$

A generalization of the considerations leading to Eq. (14) to the normalization of the cubic potential used in Ref. 22] is given in Appendix C] This generalization is somewhat non-trivial because the precise form of the strong coupling Hamiltonian depends on the normalization of the initial perturbation. When translated to the conventions of Ref. 22], the coefficient of relative order $\xi^{3}$ becomes $1 / 108$ for all states of the spectrum; this affords an explanation for the observation made originally in Table 2 of Ref. [22].

The expansion of the ground state resonance energy $E^{[q c]}(\chi)$ in powers of $\chi$ reads

$$
E^{[\mathrm{qc}]}(\chi)=\sum_{L=0}^{\infty} E_{L}^{[\mathrm{qc}]} \chi^{L} .
$$

Unlike the perturbative expansion in $g$, it is convergent in a disk. Therefore, $E(g)$ has a convergent large $g$ expansion of the form

$$
\begin{aligned}
E(g) & =g^{1 / 5}\left(-\frac{1}{3} \xi^{3}+E^{[\mathrm{qc}]}(\chi)\right) \\
& =-\frac{1}{3 g}+g^{1 / 5} \sum_{L=0}^{\infty} E_{L}^{[\mathrm{qc}]} g^{-4 L / 5} .
\end{aligned}
$$

These simple transformations show that $E^{[\mathrm{sc}]}(\xi)$ has only one term odd in $\xi$, which moreover can be read off from Eq. (20). As a consequence, for $g$ large, the relevant expansion variable is $g^{-4 / 5}$ rather than $g^{-2 / 5}$, an observation that influences the construction of optimized ODM summation methods for our problem. In the uniformizing variable $g^{-1 / 5}$, it is sufficient to determine the eigenvalues for

$$
-\frac{\pi}{4} \leq \arg \left(g^{-1 / 5}\right) \leq \frac{\pi}{4},
$$

in order to know them in the whole first Riemann sheet of the $\chi$ variable $(-\pi \leq \arg (\chi) \leq \pi)$. Conversely, knowledge of the resonance eigenvalues for $-\pi \leq \arg (\chi) \leq \pi$ implies a determination for $-5 \pi / 4 \leq \arg (g) \leq 5 \pi / 4$ which exceeds the first Riemann sheet.

Indeed, the Hamiltonian (17) is $\mathcal{P} \mathcal{T}$ symmetric for all real values of the "quadratic coupling" $\chi$ but corresponds to the Hamiltonian (10) only in the case $\chi \geq 0$. It is thus interesting to study its spectrum also in the region $\chi<0$, which corresponds to the analytic continuation $g=|g| \exp (5 \mathrm{i} \pi / 4)$. Equation (17) is also related to residues of poles of solutions of a linear system associated to the first Painlevé equation [23].

\section{GENERAL SUMMATION METHODS}

\section{A. Idea of the method}

We here briefly recall the ODM summation method. Details can be found elsewhere [13, 14]. The method is based on some a priori knowledge, or educated guess, of the analytic properties of the function that is expanded. It applies both to convergent and divergent series, although it is mainly useful in the latter situation.

In what follows, we consider a function analytic in a sector and mappings $g \mapsto \lambda$ of the form

$$
g=\rho \zeta(\lambda),
$$

where $\zeta(\lambda)$ is a real analytic function increasing on $0 \leq$ $\lambda<1$, such that $\zeta(\lambda)=\lambda+\mathcal{O}\left(\lambda^{2}\right)$ and, for $\lambda \rightarrow 1$, $\zeta(\lambda) \propto(1-\lambda)^{-\alpha}$ with $\alpha>1$. A possible choice is

$$
g=\rho \frac{\lambda}{(1-\lambda)^{\alpha}} .
$$

The parameter $\alpha$ has to be chosen in accordance with the analytic properties of the function $E$ (a rational number in our examples) and $\rho$ is an adjustable parameter, whose interpretation is an order-dependent, "artificial, local radius of convergence" of the truncated, divergent perturbative expansion (which becomes smaller as the order of the transformation is increased). An important property that singles out relevant mappings here is the following: one chooses mappings such that, for $g \rightarrow \infty$ and thus $\lambda \rightarrow 1$, the quantity $g^{-1 / \alpha}$ has a regular expansion in powers of $1-\lambda$. A more general discussion of the method can be found in Ref. [13, 14]. 
After the mapping (22), $E$ is given by a Taylor series in $\lambda$ of the form

$$
E(g(\lambda))=\sum_{L=0}^{\infty} P_{L}(\rho) \lambda^{L},
$$

where the coefficients $P_{L}(\rho)$ are polynomials of degree $L$ in $\rho$. Since the result is formally independent of the parameter $\rho$, the parameter can be chosen freely. At $\rho$ fixed, the series in $\lambda$ is still divergent, but it has been verified on a number of examples (all Borel summable), and proven in certain cases 24] that, by adjusting $\rho$ order by order, one can devise a convergent algorithm.

The $K$ th approximant $E^{(K)}(g)$ is constructed in the following way: one truncates the expansion at order $K$ and chooses $\rho$ as to cancel the last term. Since $P_{K}(\rho)$ has $K$ roots (real or complex), one chooses, in general, for $\rho$ the largest possible root (in modulus) $\rho=\rho_{K}$ with $P_{K}\left(\rho_{K}\right)=0$, for which $P_{K}^{\prime}(\rho)$ is small. This leads to a sequence of approximants $E(g) \approx E^{(K)}(g)$, where

$$
E^{(K)}(g)=\sum_{L=0}^{K} P_{L}\left(\rho_{K}\right) \lambda^{L}\left(g, \rho_{K}\right) ; \quad P_{K}\left(\rho_{K}\right)=0 .
$$

The term $P_{K+1}\left(\rho_{K}\right)$ then gives an order of magnitude of the error.

In the case of convergent series, it is expected that $\rho_{K}$ has a non-vanishing limit for $K \rightarrow \infty$. By contrast, for divergent series it is expected that $\rho_{K}$ goes to zero for large $K$ as

$$
\rho_{K}=\mathcal{O}\left(E_{K}^{-1 / K}\right) .
$$

The intuitive idea here is that $\rho_{K}$ corresponds to a 'local' radius of convergence.

Let us conclude this section with a few remarks. Above, we have used the selection criterion $P_{K}\left(\rho_{K}\right)=0$ for the order-dependent $\rho_{K}$ which are used to calculate the polynomials $P_{L}\left(\rho_{K}\right)$, for $L=0, \ldots, K-1$. Alternatively, one can choose the largest roots $\rho_{K}$ of the polynomials $P_{K}^{\prime}\left(\rho_{K}\right)=0$ for which $P_{K}$ is small. Other mixed criteria involving a combination of $P_{K}$ and $P_{K}^{\prime}$ can also be used. Indeed, one empirically observes that the approximant is not very sensitive to the precise value of $\rho_{K}$, within errors. In the ODM method, the determination of the sequence of the $\rho_{K}$ 's is the most time-consuming computational step. Indeed, once the $\rho_{K}$ are known, for each value of $g$, the calculation reduces to inverting the mapping (22) and simply summing the Taylor series in $\lambda$ to the relevant order.

\section{B. Convergence analysis}

We here give a heuristic analysis [4, 13] of the convergence of the ODM method that shows how the convergence can be optimized. This will justify the choice of the class of zeros of the polynomials $P_{K}$ or $P_{K}^{\prime}$ and provide a quantitative analysis of the corresponding convergence.

In order to focus the analysis, we assume that we are dealing with functions that have the properties of the eigenvalues of the Hamiltonian (11). We consider only real functions analytic in a cut-plane with a cut along the real negative axis and a Cauchy representation of the form

$$
E(g)=E(0)+\frac{g}{\pi} \int_{-\infty}^{0_{-}} \mathrm{d} g^{\prime} \frac{\Delta\left(g^{\prime}\right)}{g^{\prime}\left(g^{\prime}-g\right)},
$$

where the subtraction ensures the convergence of the integral for $g \rightarrow-\infty$.

For the example we discuss here, one can derive by a steepest descent calculation [7 9] an asymptotic behaviour of the form

$$
\Delta(g) \underset{g \rightarrow 0_{-}}{\propto} \frac{1}{(-g)^{b+1}} \exp \left(\frac{A}{g}\right), \quad A>0 .
$$

The function $E(g)$ can be expanded in powers of $g$,

$$
E(g)=\sum_{L=0}^{\infty} E_{L} g^{L}, \quad E_{L}=\frac{1}{\pi} \int_{-\infty}^{0_{-}} \frac{\mathrm{d} g}{g^{L+1}} \Delta(g),
$$

the Cauchy representation of $E_{L}$ being valid for $L>0$.

The asymptotic behaviour (28) then implies a large order behaviour of the form (3i),

$$
E_{L} \underset{L \rightarrow \infty}{\propto}(-A)^{-L} \Gamma(L+b+1) \underset{k \rightarrow \infty}{\propto}(-A)^{-L} L^{b} L ! .
$$

A remark is in order. When $g$ becomes negative in Eq. (7), the expression i $\sqrt{g}$ becomes real, and the corresponding resonance and antiresonance eigenvalues are attached to the upper and lower side of the integration contour which extends from $g^{\prime}=-\infty$ to $g^{\prime}=0$. The discontinuity $\Delta\left(g^{\prime}\right)$ is the difference of the imaginary parts of the resonance and antiresonance eigenvalues. For the potential we study here, it has been proved that $\Delta\left(g^{\prime}\right)$ is positive [11, and this is consistent with Eq. (28). In expression (27), for $g>0$ the integrand is positive throughout the integration contour (the denominator contains a product of two negative quantities). The eigenvalues $E(g)$ of the $\mathcal{P} \mathcal{T}$ symmetric Hamiltonian (1) thus are positive since $E(0)=1 / 2$ is positive. Similarly, the expression (29) shows that the sign of $E_{L}$ is $(-1)^{L+1}$, a result consistent with the estimate given in Eq. (30).

We now introduce the order-dependent mapping (22),

$$
g=\rho \zeta(\lambda),
$$

with the understanding that the point $\lambda=1$ corresponds to the point $g \rightarrow \infty$. Because we can alternatively formulate the dispersion relation in terms of $\lambda$, the Cauchy representation then can be written as

$$
E(g(\lambda))=\frac{1}{\pi} \oint_{\Gamma} \mathrm{d} \lambda^{\prime} \frac{\Delta\left(g\left(\lambda^{\prime}\right)\right)}{\lambda^{\prime}-\lambda},
$$




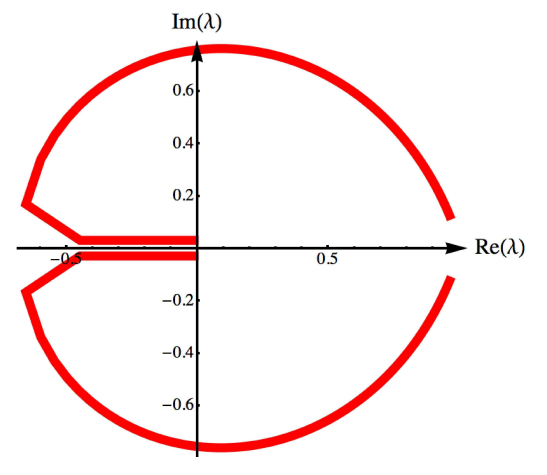

FIG. 1: (Color online.) Image in the complex $\lambda$ plane of the negative real $g$ axis under the mapping $g=\rho \lambda /(1-\lambda)^{5 / 2}$, for $\rho=1 / 2$. A point infinitesimally displaced above the negative real axis is mapped onto a point in the upper complex $\lambda$ plane.

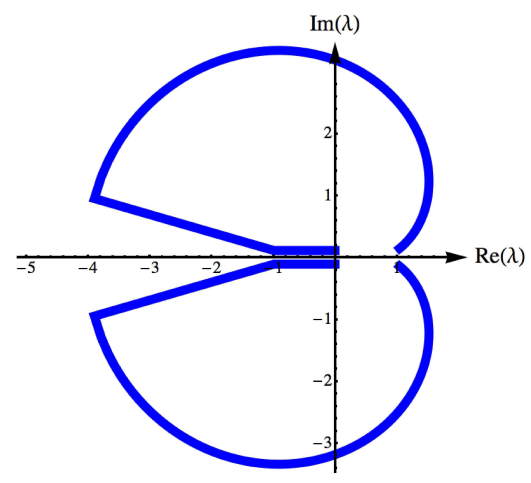

FIG. 2: (Color online.) Same as Fig. 1 but for the mapping $g=\rho \lambda /(1-\lambda)^{5 / 4}$.

where $\Gamma$ initially is the image of the cut on the real negative axis (see Fig. 1 and 2 for examples). The contour $\Gamma$ can then be deformed if the function has analyticity properties beyond the first Riemann sheet.

We expand

$$
E(g(\lambda))=\sum_{K=0}^{\infty} P_{K}(\rho)[\lambda(g)]^{K}
$$

with

$$
P_{K}(\rho)=\frac{1}{\pi} \oint_{\Gamma} \frac{\mathrm{d} \lambda}{\lambda^{K+1}} \Delta(g(\lambda)) .
$$

For $K \rightarrow \infty$, the factor $\lambda^{-K}$ favours small values of $|\lambda|$, but for too small values of $\lambda$, the exponential decay of $\Delta(g(\lambda))$ takes over. Thus, $P_{K}\left(\rho_{K}\right)$ can be evaluated by the steepest descent method. The ansatz

$$
\rho_{K} \underset{K \rightarrow \infty}{\sim} \frac{R}{K}, \quad R>0,
$$

implies that in the limit $K \rightarrow \infty$, the saddle point values of $\lambda$ are independent of $K$ and that $g(\lambda) \rightarrow 0$. The former statement can easily been seen by taking the logarithm of the integrand in Eq. (34) and observing that
$K$ factors out in the equation defining the saddle point. Thus, $\Delta(g)$ can be replaced by its asymptotic form (28) for $g \rightarrow 0_{-}$, except for $g(\lambda)$ of order one (or larger) and thus $\lambda$ close to the initial endpoint $\lambda=1$ for $K \rightarrow \infty$. The contribution of the endpoint depends on the analytic properties of $\Delta(g)$ but is bounded by a quantity of order $\exp \left(C_{1} K^{1-1 / \alpha}\right)$ [see Eq. (46)]

In what follows we set

$$
\frac{R}{A}=\mu
$$

since $\mu$ is the only parameter (and it is independent of the normalization of $g$ ). The behaviour of $P_{K}\left(\rho_{K}\right)$ is then given by the sum of leading saddle contributions. Each saddle point contribution is of order

$$
\left\{P_{k}\left(\rho_{K}\right)\right\}_{\text {saddle point }}=\mathcal{O}\left(\mathrm{e}^{K \sigma}\right), \quad \sigma \equiv \frac{1}{\mu \zeta(\lambda)}-\ln (\lambda),
$$

where $\lambda$ is determined by the saddle point equation

$$
\sigma^{\prime}(\lambda)=-\frac{1}{\mu} \frac{\zeta^{\prime}(\lambda)}{\zeta^{2}(\lambda)}-\frac{1}{\lambda}=0
$$

The analysis is simple only if $\lambda$ at the saddle point is real negative. By contrast, if $\lambda$ is complex, the analytic properties of $\Delta(g)$ and the possibility of deforming the contour $\Gamma$ become relevant.

For $\mu$ large, the equation has a unique solution $(\lambda \sim$ $-1 / \mu)$, which is real negative. Then we note that at this saddle point, as a function of $\mu$,

$$
\frac{\partial \sigma}{\partial \mu}=-\frac{1}{\mu^{2} \zeta(\lambda)}>0
$$

as long as the saddle point value of $\lambda$ is negative. This suggests decreasing $\mu$ as much as possible to improve the convergence. The exponential rate $\sigma$ corresponding to the saddle point vanishes for

$$
0=\operatorname{Re}(\sigma(\lambda))=\frac{1}{\mu \zeta(\lambda)}-\ln (-\lambda)
$$

and this defines a special value $\mu=\mu_{c}$ of the parameter $\mu$. In [4, 13, 14], we have discussed in some detail mappings of the form

$$
g=\rho \frac{\lambda}{(1-\lambda)^{\alpha}}
$$

for various values of $\alpha$. Most notably, $\alpha=5 / 2$ has been applied to the present problem (for a motivation regarding this mapping, see Appendix $\mathrm{A}$ ).

The system of Eqs. (38) and (40) can be solved for the 
variables $\mu=\mu_{c}$ and $\lambda=\lambda_{c}$. We obtain

$$
\begin{aligned}
& \text { mapping (a): } g=\rho \frac{\lambda}{(1-\lambda)^{5 / 4}}, \\
& \Rightarrow \mu_{c}^{(a)}=3.811522 \ldots, \quad \lambda_{c}^{(a)}=-0.259901 \ldots, \\
& \text { mapping (b): } g=\rho \frac{\lambda(1-\lambda / 2)}{(1-\lambda)^{5 / 2}}, \\
& \Rightarrow \mu_{c}^{(b)}=4.445762 \ldots, \quad \lambda_{c}^{(b)}=-0.216262 \ldots, \\
& \text { mapping }(\mathrm{c}): \quad g=\rho \frac{\lambda}{(1-\lambda)^{5 / 2}}, \\
& \Rightarrow \mu_{c}^{(c)}=4.895690 \ldots, \quad \lambda_{c}^{(c)}=-0.189645 \ldots
\end{aligned}
$$

Then, we expect the error $\varepsilon_{K}$ at order $K$ to be of the same order as the leading saddle point contribution, that is, of order $P_{K}\left(\rho_{K}\right) \lambda\left(g, \rho_{K}\right)^{K}$ or in view of Eq. (37),

$$
\varepsilon_{K}=\mathcal{O}\left(\mathrm{e}^{K \sigma} \lambda\left(g, \rho_{K}\right)^{K}\right) .
$$

For $K \rightarrow \infty$, at $g$ fixed, $\lambda$ goes to 1 as

$$
1-\lambda\left(g, \rho_{K}\right) \underset{K \rightarrow \infty}{\sim} C_{2}(K g)^{-1 / \alpha},
$$

where for $\mu=\mu_{c}$ and methods (42a), (42b) (42c), respectively

$$
\begin{aligned}
& C_{2}^{(a)}=\left(A \mu_{c}^{(a)}\right)^{4 / 5} \approx 10.23, \\
& C_{2}^{(b)}=\left(\frac{A \mu_{c}^{(b)}}{2}\right)^{2 / 5} \approx 3.40 \\
& C_{2}^{(c)}=\left(\frac{A \mu_{c}^{(c)}}{2}\right)^{2 / 5} \approx 3.53 .
\end{aligned}
$$

Then,

$$
\lambda^{K}\left(g, \rho_{K}\right) \underset{K \rightarrow \infty}{\sim} \exp \left(-C_{2} K^{1-1 / \alpha} g^{-1 / \alpha}\right) .
$$

Then, two cases are possible:

Case (i): if the contribution to the integral corresponding to $g(\lambda)$ of order unity decreases exponentially with $K$ (which implies the possibility of deforming the contour $\Gamma$ ), we can also choose $\mu<\mu_{c}$ and the ODM method converges for all $g \neq 0$. Moreover, this in turn implies that the function $E(g)$ is an entire function of $g^{-1 / \alpha}$. This clearly is a non-generic situation, but several examples have been met in the form of simple integrals.

Case (ii): in a generic situation where the mapping removes the singularity at infinity but other singularities are present, as explained in Refs. [4, 13, 14] we then expect the optimal mapping to correspond to $\mu=\mu_{c}$ and $P_{K}\left(\rho_{K}\right)$ (or $P_{K+1}\left(\rho_{K}\right)$ if we choose for $\rho_{K}$ a zero of $P_{K}$ ) to be, asymptotically for $K \rightarrow \infty$, itself of order

$$
P_{K}\left(\rho_{K}\right)=\mathcal{O}\left[\exp \left(C_{3} K^{1-1 / \alpha}\right)\right]
$$

where $\alpha=5 / 4$ for method (42a) and $\alpha=5 / 2$ for methods (42b) and (42c), respectively.

The domain of convergence then depends on the sign of the constant $C_{3}$. For $C_{3}>0$, the domain of convergence is

$$
|g|<\left(\frac{C_{2}}{C_{3}}\right)^{\alpha}\left[\cos \left(\frac{\arg (g)}{\alpha}\right)\right]^{\alpha} .
$$

For $\alpha=5 / 2$, this domain extends beyond the first Riemann sheet and requires analyticity of the function $E(g)$ in the corresponding domain.

For $C_{3}<0$, the domain of convergence is the union of the sector $|\arg (g)|<\pi \alpha / 2$ and the domain

$$
|g|>\left|\frac{C_{2}}{C_{3}}\right|^{\alpha}\left[-\cos \left(\frac{\arg (g)}{\alpha}\right)\right]^{\alpha} .
$$

Again for $\alpha=5 / 2$, this domain extends beyond the first Riemann sheet. We will see that this is the situation realized in the three methods (42a), (42b) and 42c).

One might now wonder how this analysis is related to the zeros of $P_{K}$ or $P_{K}^{\prime}$. Choosing the relevant zeros is a natural way of finding the region of minimal values. In fact, the relevant zeros lie in the region where two leading saddle points cancel or the leading saddle point(s) cancel the contribution coming from the remaining part of the Cauchy integral.

Indeed, in the discussed examples of case (i), one could show that the zeros correspond to a cancellation between saddle points. In the case (ii), empirically one observes that the zeros are consistent with the asymptotic behaviour (35) but, in general, with power-law corrections:

$$
\rho_{K}=\frac{R}{K}\left(1+\delta_{K}\right),
$$

where $\delta_{K} \rightarrow 0$ for $K \rightarrow \infty$. A comparison with Eq. (37) then leads to the following correction factor to the saddle point contribution

$$
\exp \left(-\frac{K \delta_{K}}{\mu_{c} \zeta\left(\lambda_{c}\right)}\right)=\exp \left(K \delta_{K} \ln \left(-\lambda_{c}\right)\right) .
$$

Consistency with the preceding analysis, cancellation of the endpoint contribution in the integral and convergence analysis, then requires

$$
\delta_{K}=\mathcal{O}\left(K^{-1 / \alpha}\right)
$$

and this is also what is generally found.

\section{OPTIMIZED SUMMATION METHODS}

\section{A. ODM summation with $\alpha=5 / 4$}

We now consider the spectrum of the Hamiltonian (11), which can be obtained by solving the time-independent Schrödinger equation

$$
\left(-\frac{1}{2} \frac{\partial^{2}}{\partial x^{2}}+\frac{1}{2} x^{2}+\frac{\mathrm{i}}{6} \sqrt{g} x^{3}\right) \psi(x)=E \psi(x)
$$


with appropriate boundary conditions.

The spectrum has a perturbative expansion in integer powers of $g$ with real (rational) coefficients alternating in sign, of the form (2), which can be derived up to large orders by standard techniques. Instanton calculus, based on a steepest descent evaluation of the corresponding path integral, allows to calculate the classical action $A$ relevant for the large order behaviour (3). One finds

$$
A=\frac{24}{5} \text {. }
$$

According to Eq. (15), the strong coupling expansion of an eigenenergy $E(g)$ of the cubic contains a coefficient $g^{1 / 5}(-1 / 3) g^{-6 / 5}=-\frac{1}{3} g^{-1}$. We can subtract this term and introduce the function

$$
F(g)=\frac{1}{3}+g E(g),
$$

which has a regular small $g$ expansion and a large $g$ expansion of the form

$$
F(g)=g^{6 / 5} E^{[\mathrm{qc}]}\left(g^{-4 / 5}\right)=g^{6 / 5} \sum_{K=0}^{\infty} E_{K}^{[\mathrm{qc}]} g^{-4 K / 5}
$$

where the $E_{K}^{[\mathrm{qc}]}$ coefficients have been defined in Eq. (19). Our aim is to investigate to which extent the strongcoupling expansion is described by an ODM summation of weak-coupling perturbation theory. To this end, according to the discussion made in Appendix A of Ref. [4], we now introduce the mapping (42a) which reads

$$
g=\rho \frac{\lambda}{(1-\lambda)^{5 / 4}}
$$

and set

$$
F(g(\lambda))=(1-\lambda)^{-3 / 2} \phi(\lambda, \rho)
$$

The function $\phi$ has a regular expansion both at $\lambda=0$ $(g=0)$ and $\lambda=1(g \rightarrow \infty)$. The analysis of Sec. III then yields $\mu_{c}^{(a)}=3.811522 \ldots$ and thus $R=A \mu_{c}^{(a)}=$ $18.295306 \ldots$ in the notation of Sec. IIIB [see Eq. (42a)].

Although the summation methods apply to all eigenvalues, from now on we consider only the ground state energy. The first terms of its perturbative expansion are

$$
E(g)=\frac{1}{2}+\frac{11}{288} g-\frac{930}{(288)^{2}} g^{2}+\mathcal{O}\left(g^{3}\right) .
$$

The convergence analysis of Ref. [4], partially reproduced in Sec. III. clearly indicates that in this problem, one expects a convergence of the ODM method only in $\mathrm{e}^{-C K^{\zeta}}$ with $\zeta<1$, and this is confirmed here. We find strong evidence for a finite distance singularity. It is consistent with this property that we can fit the zeros $\rho_{K}$ of $P_{K}^{\prime}(\rho)$ using the functional form

$$
\rho_{K}=\frac{A \mu_{c}^{(a)}}{K}\left(1-\frac{12.94}{(K+3)^{4 / 5}+11.97}\right) .
$$

The zeros of $P_{k}$ can be fitted by an analogous formula but with slightly different coefficients for the correction term. It has proven convenient to use the latter expression in the ODM summation in the place of the exact values.

There is strong numerical evidence for a singularity at $g \approx 0.687 \exp (5 \mathrm{i} \pi / 4)$. (see Sec. VIIB). One thus expects the ODM approximants of order $K$ to converge to the energy eigenvalues as (see Eq. (45a))

$$
\text { constant } \times \exp \left\{\left[-13.8-10.23 \operatorname{Re}\left(g^{-4 / 5}\right)\right] K^{1 / 5}\right\},
$$

consistent with a domain of convergence

$$
\operatorname{Re}\left(g^{-4 / 5}\right)=\operatorname{Re} \chi>-1.351 \text {. }
$$

Indeed, this expression reproduces well the region where the convergence factor becomes close to unity. The domain of convergence contains a segment on the real negative $\chi$ axis not contained in the convergence domain obtained by the ODM method (42c) of Ref. [4], which is

$$
\operatorname{Re}\left(g^{-2 / 5}\right)=\operatorname{Re} \sqrt{\chi}>-0.0826 \text {. }
$$

The expression (51) combined with a fit of the relevant zeros of the polynomials $P_{K}^{\prime}$ yields a coefficient of about 17.4 instead of 13.8 in Eq. (61). Finally, a rough numerical analysis of the convergence toward $E_{0}^{[\mathrm{qc}]}$ rather yields $\exp \left(-18 K^{1 / 5}\right)$. This implies that either the asymptotic regime is reached very slowly, and this is the most likely explanation, or our heuristic convergence analysis in this particular example is oversimplified. This problem requires a further analysis.

Compared with the ODM method of Ref. [4], the convergence is expected here to be slower at small $g$ and, eventually, at extremely large $K$. At order 150 , for $g$ real and positive, the transition occurs for $|g|$ slightly larger than 1 . Of course, the method with $\alpha=5 / 2$ also converges in a larger sector, up to $|\arg (g)|=5 \pi / 4$ instead of $|\arg (g)|=5 \pi / 8$ for $\alpha=5 / 4$.

Finally, because the convergence is smooth (unlike what happens with $\alpha=5 / 2$ methods) the precision of the results can still be improved (see Tables 【. II) by using repeatedly the algorithm that to any sequence $S_{n}$ substitutes

$$
S_{n} \mapsto \frac{S_{n} S_{n+2}-S_{n+1}^{2}}{S_{n}+S_{n+2}-2 S_{n+1}} .
$$

Empirically, we find that it is most advantageous to apply this method to the odd and even orders separately. This is a variant of Aitken's $\Delta^{2}$ process as it is known in the literature. Then at order 150, the method (42a) remains the most efficient one for large coupling $|g| \geq 1$.

\section{B. ODM summation with $\alpha=5 / 2$}

In Ref. [4], the ODM method with the mapping (42c),

$$
g=\frac{\lambda}{(1-\lambda)^{5 / 2}},
$$


was applied directly to the energy eigenvalue $E(g)$, which has a large-order expansion of the form

$$
E(g)=g^{1 / 5} \sum_{K=0}^{\infty} E_{K}^{[\mathrm{scc}]} g^{-2 k / 5} .
$$

This expansion effectively becomes a large-order expansion in powers of $g^{-4 / 5}$ if one subtracts the term of order $-\frac{1}{3} g^{-6 / 5}$. The latter is the only term not accounted for by the powers of $g^{-4 / 5}$.

The numerical results in Sec. $\nabla$ justify the introduction of the function $F(g)$, which is defined in Eq. (56), and the mapping (42a) that precisely takes the special form of the large $g$ expansion into account. However, while the method (42a) is more efficient for $g$ large, the method (42C) yields more precise results for $K$ very large and converges in a larger sector of the complex plane.

It is thus natural to investigate an ODM method, still with $\alpha=5 / 2$, that incorporates in a better way the properties of the large $g$ expansion than the method given by Eq. (42C). To this end, we again introduce the function (55) and modify the form of the mapping to transfer the property of even powers of $g^{-2 / 5}$ into even powers of $1-\lambda$. A possible choice for a modified mapping is the mapping (42b),

$$
\begin{aligned}
g & =\rho \frac{\lambda(1-\lambda / 2)}{(1-\lambda)^{5 / 2}} \\
\Rightarrow \quad\left(\frac{2 g}{\rho}\right)^{-2 / 5} & =(1-\lambda)\left[1-(1-\lambda)^{2}\right]^{-2 / 5} .
\end{aligned}
$$

Also,

$$
(1-\lambda)^{-3}=\left(\frac{2 g}{\rho}\right)^{6 / 5}\left[1-(1-\lambda)^{2}\right]^{-6 / 5} .
$$

With this mapping, the function

$$
\phi(\lambda)=(1-\lambda)^{3} F(g(\lambda))
$$

is indeed only a function of $(1-\lambda)^{2}$. However, in contrast to the previous mapping, the symmetry $g^{-2 / 5} \mapsto-g^{-2 / 5}$ is only implemented approximatively, at a finite order, because the series in powers of $\lambda$ is truncated.

Since one cannot expect geometric convergence of the ODM method defined by Eq. (42b), the relevant value of $\mu$ is $\mu_{c}^{(b)}=4.445762607 \ldots$ (see Sec. IIIB). It follows that $C_{2}^{(b)}=(R / 2)^{2 / 5}=\left(A \mu_{c}^{(b)} / 2\right)^{2 / 5}=3.401535 \ldots$ [see Eq. 445b].

For the ground state energy, we empirically find that the location of the zeros of $P_{K}^{\prime}$ can be fitted by

$$
\rho_{K}=\frac{A \mu_{c}}{K}\left(1-\frac{5.0}{(K+2)^{4 / 5}+4.6}\right) .
$$

One also expects a term decreasing only like $K^{-2 / 5}$ but its coefficient is apparently too small to be detected compared to the larger $K^{-4 / 5}$ contribution. The same remark actually applies to the method (42C). We have not compared the convergence of methods (42b) to method (42C) systematically, but methods (42b) is definitively better for $g \rightarrow \infty$ than with the initial $\alpha=5 / 2$ mapping of Eq. (42C) (see Tables \and at order 55, with the new mapping (42b), one finds the value $E_{0}^{[\mathrm{qc}]}=0.372545$ (9) for the leading strong-coupling coefficient defined according to Eq. (19), corresponding to a relative error of the order of $10^{-7}$, that is, two order of magnitudes better as compared to the mapping (42c). At order 150, one finally obtains $E_{0}^{[\mathrm{qc}]}=0.37254578(6)$, a value that differs by $4 \times 10^{-9}$ from the strong coupling limit in (92).

Values of the ground state energy $E(g)$ for various real, positive and negative values of $g$, and order 55 and 150 , are reported in Tables IIV

The general conclusion is: at the order we calculate, as expected, the results for the modified method $42 \mathrm{~b}$ are more precise than for the more straightforward method (42C) if $g$ is large, but comparable or worse for $g$ small. However, for $|g| \geq 1$, results given by the method (42a) with $\alpha=5 / 4$ are always the best. This is consistent with the fact that higher-order corrections of the form $g^{-4 K / 5}$ are well represented by the corresponding expansion for $E^{[\mathrm{qc}]}(g)$. Note that the correction of relative order $g^{-6 / 5}$ for $E^{[s c]}(g)$ provides for a certain irregularity in the expansion (13). As a conclusion, the advantages of the modified version $42 \mathrm{~b}$ seem to be somewhat limited, and we have not investigated the method more thoroughly, but we have included the results in the tables to stress the general consistency of the different implementations of the ODM summation methods.

\section{NUMERICAL RESULTS: GROUND STATE ENERGY}

We first display a few typical results obtained for $g$ finite, positive and negative, by the various methods we discuss in this article: the ODM method with $\alpha=5 / 4$, the methods of Sec. IVB and Ref. [4] and, finally, the continued fraction of Sec. VIIB below.

\section{A. Real positive axis}

For $g$ finite and real positive, we have chosen the same values as in Ref. [4] and added results for the case $g=0.5$. Most results are displayed in Table 1 for the results at order 55, to allow for a direct comparison with [4] and give an idea about the rate of convergence, and in Table at order 150 . We use everywhere the convention that the numerical uncertainty applies to the digit in parentheses (this may also affect the preceding digit by a shift \pm 1 ).

For $g=0.5$, at order 150, with the ODM method given 
TABLE I: The ODM mappings given in Eq. 442a, 42b and 42c) are compared with respect to their convergence properties on the real positive axis at a transformation order of 55. Method (42a) with $\alpha=5 / 4$ and extrapolation clearly provides the best answers for large and moderate coupling. The modified method (42b) with $\alpha=5 / 2$ interpolates between method 42a and (42c); indeed, the application of the straightforward ODM method (42c) still provides for the best convergence at weak coupling.

\begin{tabular}{cll}
\hline \hline$g$ & 0.5 & \multicolumn{1}{c}{1} \\
\hline Method of Eq. (42a) & $0.516891764253171978211158895(6)$ & $0.53078175930417667113556(1)$ \\
Method of Eq. (42b) & $0.5168917642531719782111588(9)$ & $0.53078175930417667113(4)$ \\
Method of Eq. (42c) & $0.51689176425317197821115889(6)$ & $0.530781759304176671135(6)$ \\
\hline \hline$g$ & 5.0 & 21.6 \\
\hline Method of Eq. (42a) & $0.60168393320519196159(1)$ & $0.73340992048542796(4)$ \\
Method of Eq. (42b) & $0.601683933205(2)$ & $0.733409920(5)$ \\
Method of Eq. (42c) & $0.601683933205(2)$ & $0.73340992(1)$ \\
\hline \hline
\end{tabular}

TABLE II: Same as Table@ but for a transformation order of 150. For weak and moderate coupling, the method (42c) now takes over. For strong coupling, the convergence of method 42a with $\alpha=5 / 4$ clearly is superior to that of the other ODM methods. For completeness, we give also the results obtained from the simple continued fraction method (99). For the method defined by (42a), the numerical results are post-accelerated using the algorithm (64).

\begin{tabular}{ccc}
\hline \hline$g$ & \multicolumn{1}{c}{0.5} & \multicolumn{1}{c}{1.0} \\
\hline Method of Eq. (42a) & 0.51689176425317197821115889 & 0.530781759304176671135561818032 \\
Method of Eq. (42b) & 0.51689176425317197821115889 & 0.530781759304176671135561818032 \\
Method of Eq. [42c) & 0.51689176425317197821115889 & 0.530781759304176671135561818032 \\
Continued fraction & $0.516891764253171978211(0)$ & $0.5307817593041766711355(7)$ \\
\hline \hline$g$ & \multicolumn{1}{c}{5.0} & 21.6 \\
\hline Method of Eq. (42a) & $0.601683933205191961589356494(4)$ & $0.7334099204854279645924020(0)$ \\
Method of Eq. (42b) & $0.60168393320519196159(0)$ & $0.73340992048542(8)$ \\
Method of Eq. (42c) & $0.60168393320519196158(9)$ & $0.7334099204854(3)$ \\
Continued fraction & $0.60168393320519196158936(0)$ & $0.73340992048542796459240(3)$ \\
\hline \hline
\end{tabular}

in Eq. (42b), one finds

$$
\begin{gathered}
E(0.5)=0.5168917642531719782111588 \\
956621775(8),
\end{gathered}
$$

and with the method given in Eq. (42c)

$$
\begin{array}{r}
E(0.5)=0.5168917642531719782111588 \\
95662177609999961207(4),
\end{array}
$$

the error being in agreement with the estimate given in Ref. [4]. Finally, with the method given Eq. 42a one finds

$$
\begin{gathered}
E(0.5)=0.5168917642531719782111588 \\
956621775(8)
\end{gathered}
$$

and after extrapolation using the method (64),

$$
\begin{gathered}
E(0.5)=0.5168917642531719782111588 \\
956621776099999612(1) .
\end{gathered}
$$

For $g=1$, from a numerical solution of the Schrödinger equation one obtains

$$
\begin{gathered}
E(1.0)=0.53078175930417667113 \\
5561818032225 .
\end{gathered}
$$

At order 150, with the mapping (42c), the result reads

$$
\begin{array}{r}
E(1.0)=0.53078175930417667113 \\
556181803222595(1) .
\end{array}
$$


At order 150, the method 42b yields a result with an error of $10^{-33}$, which is slightly less precise. The method (42a) together with extrapolation (64) yields

$$
\begin{aligned}
E(1.0)= & 0.530781759304176671135561818032 \\
& 225951(1) .
\end{aligned}
$$

Clearly, for small values of $g$, that is, $g<1$, at order 150 the method of [4] gives the most precise results. The entries for $E(1.0)$ exceed the maximum number of columns available in Table Ir for results obtained at transformation order 55, see Table 【

Finally, to compare with the Padé summation of [12], we give results for $g=288 / 49$, which corresponds to $\lambda^{2}=1 / 7$ in [12]. In Ref. [12], at order 192, the reported result is $E=0.612738106388986$. In Ref. [4], using the method (42c), at order 55, one finds

$$
E\left(\frac{288}{49}\right)=0.612738106389(1) .
$$

With $\alpha=5 / 4$, using method (42a) and convergence acceleration, at order 55 , one obtains

$$
E\left(\frac{288}{49}\right)=0.612738106388984124(7) .
$$

This improves over the ODM method (42c) in Ref. [4] by six orders of magnitude. Then, at order 150, the result becomes

$$
E\left(\frac{288}{49}\right)=0.612738106388984124762089552(6) .
$$

With the method (42c), at the same order 150, one obtains

$$
E\left(\frac{288}{49}\right)=0.61273810638898412476(3) .
$$

We have also verified that

$$
E\left(\frac{288}{49}\right)=0.61273810638898412476 \ldots
$$

by a numerical solution of the Schrödinger equation.

The general conclusion is: for values $g \leq 1$, at order 150 the method with the mapping (42C) of Ref. [4] is the most precise, and the advantage increases with increasing order and decreasing parameter $g$. By contrast, for $g>1$, at least up to order 150 , the method with $\alpha=5 / 4$ takes over. Note that from now on and, in particular in the tables, we only quote the results obtained by the method (42a) after extrapolation.

Finally, the continued fraction of Sec. VIIB constructed from the strong coupling expansion (but which incorporates additional information about level merging), provides a rather good representation of the function in a wide domain.

For completeness, in the spirit of reference [12], we give some indication about the summation of the perturbative series by a continued fraction expansion. We define (for the ground state)

$$
h_{0}(g)=E(g) / E(0),
$$

and introduce the relation

$$
h_{p-1}(g)=1+\frac{\kappa_{p} g}{h_{p}(g)}, \quad h_{p}(0)=1,
$$

which allows calculating $h_{p}$ from $h_{p-1}$ and determining recursively the coefficients $\kappa_{p}$. The truncated continued fraction (obtained by replacing, at some order $p$, $h_{p}(g)$ by 1$)$ generates, alternatively, $[n+1 / n]$ and $[n / n]$ Padé approximants. For a Stieltjes function, all coefficients $\kappa_{p}$ are positive. Moreover, for a divergent series with a large order behaviour of the form (3) one expects the coefficients $\kappa_{p}$ to grow asymptotically linearly with $p$ and the error for the continued fraction truncated at order $p$ to behave like $\exp (-C \sqrt{p / g})$. This is indeed what is observed. The coefficient of the linear term is compatible with $5 / 48$. More precisely, a $\operatorname{good}$ fit is $\kappa_{p}=\left(10 p+3 \times(-1)^{p}\right) / 96+\mathcal{O}(1 / \sqrt{p})$ for $p \rightarrow \infty$. Also, the coefficient of $\sqrt{p / g}$ extrapolates to $C=2 \sqrt{48 / 5}=6.19 \ldots$ with a good precision. Compared to the various ODM summations, the convergence is limited to the first Riemann sheet, is poorer than the ODM method with $\alpha=5 / 2$ for $g$ small and is much poorer than the method with $\alpha=5 / 4$ for $g$ large. For example, at order 150 , for $g=0.5$ the error is about $1.5 \times 10^{-42}$, for $g=1$ about $6 \times 10^{-31}$, for $g=5$ about $10^{-14}$ and about $2 \times 10^{-7}$ for $g=21.6$.

\section{B. Negative real axis}

Results for four values of $g<0$ are displayed in Tables [II] and IV. From the numerical evidence, we conclude that ODM methods with $\alpha=5 / 2$ converge also for $g<0$ but, from the theoretical analysis, the convergence is expected to be poorer than for $g>0$, in agreement with the data. The ODM method (42b) with $\alpha=5 / 4$ converges also on the real negative axis for $|g|$ large enough, that is, $|g|>0.53$ approximately.

From the analysis of Ref. [4], we know that the imaginary part itself is an analytic function with singularities on the real negative axis only at $g=0$ and at infinity. Moreover, it is a simple positive decreasing function. Compared with the method with $\alpha=5 / 2$, the positivity of the imaginary part for $g=-|g|+\mathrm{i} 0$ can again be verified, but with higher precision for $g$ large. For $|g| \rightarrow \infty$ and $\operatorname{Im}(g)=0_{+}$, from the leading term in Eq. (92) suitably rotated into the complex plane, one now infers

$$
\begin{gathered}
E(g) /|g|^{1 / 5} \underset{g \rightarrow-\infty}{=} 0.3013958756586835717823(7) \\
+0.2189769214314493762936(0) \mathrm{i},
\end{gathered}
$$

a result that uses the perturbative series up order 150 .

For $g=-0.5$, the method (42b) with $\alpha=5 / 4$ is not expected to converge and, indeed, numerical indications are that this is the case. The result is somewhat equivalent to a direct summation of the initial asymptotic series, with an error of the order of the imaginary part 
TABLE III: On the real negative axis, at order 150, the real part Re $E(g)$ is approximated by several variants of ODM methods. The straightforward ODM method (42c) provides the best results for weak coupling, whereas method (42a) with $\alpha=5 / 4$, post-accelerated, is superior in the moderate and strong coupling domain. Note that the simple continued fraction method (99) also provides for a well converged answer.

\begin{tabular}{|c|c|c|}
\hline$-g$ & 0.5 & 1.0 \\
\hline Method of Eq. 42a & $0.476427408(3)$ & $0.442520045124688(4)$ \\
\hline Method of Eq. 42b & $0.47642740832(7)$ & $0.44252004512(4)$ \\
\hline Method of Eq. (42c) & $0.476427408327179(5)$ & $0.442520045124(7)$ \\
\hline Continued fraction & $0.4764274083271(9)$ & $0.4425200451246883662(3)$ \\
\hline$-g$ & 5.0 & 21.6 \\
\hline Method of Eq. 42a & $0.433890667810363128131(1)$ & $0.55405351846101380317898(0)$ \\
\hline Method of Eq. 42b & $0.4338906678(1)$ & $0.554053518(5)$ \\
\hline Method of Eq. 42c & $0.433890667(9)$ & $0.5540535(2)$ \\
\hline Continued fraction & $0.43389066781036312813116(9)$ & $0.55405351846101380317898(0)$ \\
\hline
\end{tabular}

TABLE IV: The imaginary part $\operatorname{Im} E(g)$ at transformation order 150 shows the same characteristics as the real part discussed in Table III.

\begin{tabular}{cll}
\hline \hline \multicolumn{1}{c}{$-g$} & \multicolumn{1}{c}{0.5} & \multicolumn{1}{c}{1.0} \\
\hline Method of Eq. 42a) & $0.000266662(1)$ & $0.01551792582059(4)$ \\
Method of Eq. [42b) & $0.00026666188(2)$ & $0.01551792582(0)$ \\
Method of Eq. [42c) & $0.000266661882408(1)$ & $0.015517925820(6)$ \\
Continued fraction & $0.0002666618824(6)$ & $0.0155179258205942572(2)$ \\
\hline \hline$-g$ & \multicolumn{1}{c}{5.0} & \multicolumn{1}{c}{21.6} \\
\hline Method of Eq. 42a & $0.183858086186171172893(3)$ & $0.35140177759369193624451(6)$ \\
Method of Eq. [42b) & $0.1838580861(9)$ & $0.351401777(6)$ \\
Method of Eq. (42c) & $0.183858086(0)$ & $0.3514018(0)$ \\
Continued fraction & $0.1838580861861711728933(1)$ & $0.3514017775936919362445(1)$ \\
\hline \hline
\end{tabular}

on the cut. However, compared to the direct summation of the series, in some range the successive results first oscillate around the exact value with the order $K$ with a rather slowing increasing amplitude rather than blowing up. Otherwise, we notice a situation comparable to the real positive axis. For $|g|<1$, the ODM method of [4] yields the most precise results, while for $|g| \geq 1$, the method 42a with $\alpha=5 / 4$ and convergence acceleration takes over. However, the continued fraction of Sec.VIIB gives the most precise results in the intermediate range and is equivalent for $|g|$ large.

\section{STRONG COUPLING EXPANSION AND LEVEL CROSSING}

We here demonstrate that considerable information on subleading corrections to the strong coupling asymptotics can be obtained by investigating the ODM resummed weak-coupling expansion of the energy levels.

In order to explore more thoroughly the strong coupling expansion and the analytic properties of the ground state energy of the cubic Hamiltonian, it is very useful to also consider the linear coupling Hamiltonian (17),

$$
H^{[\mathrm{qc}]}=-\frac{1}{2} \frac{\partial^{2}}{\partial x^{2}}+\mathrm{i}\left(\frac{1}{6} x^{3}+\frac{1}{2} \chi x\right),
$$


because a perturbative expansion in the variable

$$
\chi=g^{-4 / 5},
$$

of the energy level of the Hamiltonian $H^{[\mathrm{qc}]}$, defined in Eq. (17), is equivalent to a large $g$ expansion of the corresponding energy of the Hamiltonian (11). We recall here that the two Hamiltonians are only equivalent for $\chi>0$, the negative $\chi$ axis corresponding to $\arg (g)=5 \pi / 4$.

For real $g$, the ground state energy $E(g)$ of the Hamiltonian (11) is a real analytic function. From the numerical evidence provided by the ODM summation in Ref. [4], we conjecture that, that, in the variable $g^{-1 / 5}$, the eigenvalues can be continued up to $\left|\arg \left(g^{-1 / 5}\right)\right|=\pi / 4$. This implies that in the variable $\chi$, the eigenvalues $E^{[\mathrm{qc}]}(\chi)$ are real analytic with singularities only on the negative real axis. The series in powers of $\chi$ are convergent in a disk. In the case of the ground state, at the singularity nearest to the origin (numerical results indicate $\left.\chi=\chi_{c}=-1.3510 \ldots\right)$. Note that, by contrast with a Hermitian Hamiltonian, $\mathcal{P} \mathcal{T}$ symmetric Hamiltonians do not experience eigenvalue repulsion, which explains why it is possible for eigenvalues to merge for $\chi$ real.

\section{A. Strong coupling expansion}

We now concentrate on the behaviour of $E(g)$ for $g \rightarrow$ $\infty$, or, equivalently, on the small $\chi$ expansion given in Eq. (19) of $E^{[\mathrm{qc}]}(\chi)$ :

$$
E^{[\mathrm{qc}]}(\chi)=\sum_{K} E_{K}^{[\mathrm{qc}]} \chi^{K} .
$$

First, we have calculated the values of the coefficients of the large coupling expansion as determined by the ODM method (42a) with $\alpha=5 / 4$, with the improvement by the algorithm (64). The successive coefficients of the small $\chi$ expansion are related to $\phi(\lambda, \rho)$ and its derivatives taken at $\lambda=1$ (see Appendix $\mathrm{A}$ for the first terms). At leading order, one finds

$$
\phi(1, \rho)=\rho^{1 / 5} E_{0}^{[\mathrm{qc}]} .
$$

For the leading term, we obtain

$$
E_{0}^{[\mathrm{qc}]}=0.372545790452207098250601(1)
$$

Note that even at order 150, with the ODM method (42c) for the coefficient $E_{0}^{[\mathrm{qc}]}$ one still finds an uncertainty of $4.0 \times 10^{-6}$. With the method (42b), one obtains $E_{0}^{[\mathrm{qc}]}=$ $0.37254578(9)$.

From the ODM method 42a with $\alpha=5 / 4$ applied to the perturbative expansion of the function (56), at order 150, we have then determined, using the algorithm described in Appendix B, with decreasing relative precision (about $10^{-6}$ for the last term), all terms up to order $K=20$. The analysis of the behaviour of the coefficients with increasing order strongly suggests the existence of a square root singularity located at $\chi=\chi_{c}=-1.351 \pm 0.002$. A square root singularity is consistent with the existence of a level merging. To confirm this analytic structure and locate the singularity more precisely, we have assumed that the point $\chi_{c}$ corresponds to a merging between the ground state energy and the first excited state energy.

\section{B. Level merging and strong coupling expansion}

In addition to the ground state, as described, we have also generated the perturbative series for the first excited state. We do not report here the details of the numerical study of the energy of the first excited state. The behaviour of the series with respect to the ODM summation methods is very similar. The coefficients of the strong coupling expansion can be determined with comparable precision. The convergence properties of the ODM method (42c) indicate that, again, the eigenvalue is analytic in a cut-plane and that the first singularity corresponds to the level merging with the ground state energy.

We have then formed the two symmetric combinations, the half sum $S_{01}$ and the half difference squared $\Delta_{01}$ (GS and ES stand for ground state and first excited state, respectively):

$$
\Delta_{01}=\frac{1}{4}\left(E_{\mathrm{GS}}^{[\mathrm{qc}]}-E_{\mathrm{ES}}^{[\mathrm{qc}]}\right)^{2}, \quad S_{01}=\frac{1}{2}\left(E_{\mathrm{GS}}^{[\mathrm{qc}]}+E_{\mathrm{ES}}^{[\mathrm{qc}]}\right) .
$$

If the ansatz is correct, the two functions are not singular at $\chi_{c}$ and $\Delta_{01}$ must vanish linearly at $\chi_{c}$. This is indeed what is observed (see Figs. 3 and 4). The result confirms that at $\chi_{c}$ the eigenvalues corresponding to the ground state and the first excited state, merge and for $\chi<\chi_{c}$ become complex conjugate.

More precisely, the direct summation of the series for $\Delta_{01}$ with the ODM method of Sec. IVA shows that $\Delta_{01}$ vanishes linearly with $\chi$ at the point

$$
\chi_{c}=-1.3510415966(3),
$$

a result fully consistent with the direct study of the ground state. At this point, $E^{[\mathrm{qc}]}\left(\chi_{c}\right)=$ $0.41330579447(3)$. As a by-product, one also obtains for the ground state, the value of $E^{[\mathrm{qc}]}\left(\chi=-2^{4 / 5}\right)=$ $0.38985020(5)-0.364427(9) \mathrm{i}$, which confirms the result coming from the continued fraction in Table VI.

By the same method as for the ground state, we have determined the strong coupling expansions of $S_{01}$ and $\Delta_{01}$. From these strong coupling expansions, one can recalculate the strong coupling expansion of the ground state energy. The results are completely consistent with those from the direct expansion. However, the precision is improved for the higher order coefficients as expected since the singularity at $\chi_{c}$ is now explicitly generated.

As a necessary ingredient for a more precise determination of the coefficients of the continued fraction of 
Sec. VIIB for the ground state, we have calculated the strong-coupling expansions of the difference $\Delta_{01}$ and the sum $S_{01}$ up to to order 28 . We have then inferred the strong coupling expansion of the ground state at the same order although we here give only the first 20 terms. These are useful for reference purposes and read as follows:

$$
\begin{aligned}
& E^{[\mathrm{qc}]}(\chi)=0.3725457904522070982506011(5) \\
& +0.3675358055441936035304(6) \chi \\
& +0.1437877004150665158339(0) \chi^{2} \\
& -0.0265861056270593871352(9) \chi^{3} \\
& +0.0098871650792008872905(5) \chi^{4} \\
& -0.004610019293623151602(3) \chi^{5} \\
& +0.002409342635048475211(7) \chi^{6} \\
& -0.00134885152931985498(8) \chi^{7} \\
& +0.00079061197681697837(2) \chi^{8} \\
& -0.0004788478414145725(4) \chi^{9} \\
& +0.0002972375584267145(5) \chi^{10} \\
& -0.000188065795326713(9) \chi^{11} \\
& +0.000120825549560587(6) \chi^{12} \\
& -7.8604558627946(5) \times 10^{-5} \chi^{13} \\
& +5.1674464642199(1) \times 10^{-5} \chi^{14} \\
& -3.4272947828030(3) \times 10^{-5} \chi^{15} \\
& +2.290502086987(5) \times 10^{-5} \chi^{16} \\
& -1.540915921976(3) \times 10^{-5} \chi^{17} \\
& +1.0426603452042(4) \times 10^{-5} \chi^{18} \\
& -0.709138753556(4) \times 10^{-5} \chi^{19} \\
& +0.484508166066(0) \times 10^{-5} \chi^{20}+\mathcal{O}\left(\chi^{21}\right) .
\end{aligned}
$$

Note that the errors are strongly correlated.

This expansion is also consistent with the 20 first coefficients reported in Ref. 15], which have been determined with a $10^{-10}-10^{-11}$ relative precision by a numerical solution of the eigenvalue equation. It is also consistent with results obtained for the few first terms from a numerical solution of the Schrödinger equation [16], which have relative errors of order $10^{-9}$.

\section{CONTINUED FRACTION AND ANALYTIC PROPERTIES}

\section{A. General remarks}

To discuss more thoroughly the analytic properties of the ground state energy, it is convenient to consider the Hamiltonian (17) and parameterize the energy in terms of the coupling $\chi$.

Inverting the relations (11) and (19), one finds a form relevant for the large $\chi$ behaviour of $E^{[\mathrm{qc}]}(\chi)$ :

$$
E^{[\mathrm{qc}]}(\chi)=\frac{1}{3} \chi^{3 / 2}+\chi^{1 / 4} E\left(\chi^{-5 / 4}\right) .
$$

The perturbative expansion of $E(g)$ in $g$ translates into a large $\chi$ expansion, because large $\chi$ corresponds to weak coupling $g$,

$$
\begin{aligned}
\chi^{1 / 4} E\left(\chi^{-5 / 4}\right)= & \frac{1}{2} \chi^{1 / 4}+\frac{11}{288} \chi^{-1} \\
& -\frac{155}{13824} \chi^{-9 / 4}+\cdots
\end{aligned}
$$

In particular, the imaginary part for $\chi=-|\chi|+\mathrm{i} 0$ has the expansion

$$
\begin{aligned}
\operatorname{Im} E^{[\mathrm{qc}]}(\chi)= & -\frac{1}{3}|\chi|^{3 / 2}+\frac{1}{2 \sqrt{2}}|\chi|^{1 / 4} \\
& +\frac{155}{13824 \sqrt{2}}|\chi|^{-9 / 4}+\cdots
\end{aligned}
$$

For $|\chi| \gg 1$, the imaginary part is clearly negative. The question that arises is its sign on the cut for $|\chi|$ of order unity.

Note that the large $\chi$ behaviour (93) implies that the Cauchy representation for the perturbative expansion coefficient $E_{K}^{[\mathrm{qc}]}$ can be written as

$$
E_{K}^{[\mathrm{qc}]}=\frac{1}{\pi} \int_{-\infty}^{\chi_{c}} \frac{\operatorname{Im} E^{[\mathrm{qc}]}(\chi)}{\chi^{K+1}} \mathrm{~d} \chi
$$

converges only for $K>1$. The conjecture $\operatorname{Im} E^{[\mathrm{qc}]}(\chi) \leq$ 0 , which will be substantiated in Sec. VIIB, is thus consistent with the signs of coefficients of the expansion (92). Finally, some additional information can be obtained directly from the ODM method with $\alpha=5 / 2$ (see Table VI and also by summing the small $\chi$ expansion (92) as we will show.

\section{B. Continued fraction}

To continue the expansion (92) outside the circle of convergence, we introduce the continued fraction expansion of

$$
\tilde{E}^{[\mathrm{qc}]}(\chi)=\frac{E^{[\mathrm{qc}]}(\chi)-E^{[\mathrm{qc}]}(0)}{E^{[\mathrm{qc}]^{\prime}}(0) \chi}
$$

motivated by the prejudice that $\tilde{E}^{[q c]}$ is a Stieltjes function since

$$
\operatorname{Im} \tilde{E}^{[\mathrm{qc}]}(\chi)=\frac{\operatorname{Im} E^{[\mathrm{qc}]}(\chi)}{E^{[\mathrm{qc}]^{\prime}}(0) \chi}
$$

is positive if $\operatorname{Im} E_{0}^{[\mathrm{qc}]}$ is negative and $\tilde{E}^{[\mathrm{qc}]}$ behaves like $\chi^{1 / 2}$ for $|\chi| \rightarrow \infty$. We then construct the continued 
TABLE V: Coefficients $a_{p}$ of the continued fraction for $K=150$. The singularity is consistent with a limit $0.1850424 \ldots$

\begin{tabular}{llll}
\hline \hline$p=1$ & $p=2$ & $p=3$ & $p=4$ \\
0.39122093207263598993 & 0.18489832962286956168 & 0.18699386165533376095 & 0.18768406689188109149 \\
\hline$p=5$ & $p=6$ & $p=7$ & $p=8$ \\
$0.1851910686950779010(9)$ & $0.184774976141944774(1)$ & $0.18470222481783684(5)$ & $0.1850738286033382(9)$ \\
\hline$p=9$ & $p=10$ & $p=11$ & $p=12$ \\
$0.185134240394894(8)$ & $0.18510363919575(7)$ & $0.1849998937209(1)$ & $0.185015894534(4)$ \\
\hline$p=13$ & $p=14$ & $p=15$ & $p=16$ \\
$0.18504172330(4)$ & $0.18506432329(6)$ & $0.0 .1850419101(5)$ & $0.1850366878(1)$ \\
\hline \hline
\end{tabular}

fraction expansion for the function $\tilde{E}^{[\mathrm{qc}]}$ by introducing the recursion relation

$$
f_{p-1}(\chi)=1+\frac{a_{p} \chi}{f_{p}(\chi)}, \quad f_{p}(0)=1
$$

with the initial condition $f_{0}(\chi)=\tilde{E}^{[\mathrm{qc}]}(\chi)$. It allows us to calculate the coefficients $a_{p}$ recursively. The truncated continued fractions, obtained by replacing at some order $f_{p}(\chi)$ by 1 , generate, alternatively, $[n+1 / n]$ and $[n / n]$ Padé approximants, depending on whether $p$ is odd or even.

For a Stieltjes function, all coefficients $a_{p}$ are positive. Indeed, using the coefficients (92), which are obtained from the series at order 150 , we find $a_{p}>0$ (see Table V) for $p \leq 27$ and the coefficients seem to converge slowly, within errors, toward the limit expected for a square root singularity at $\chi=\chi_{c}: 1 /\left(4 \chi_{c}\right)=-0.18504241514 \ldots$. For example, the two last coefficients determined with sufficient precision are $a_{24}=0.1850464(8)$ and $a_{25}=$ $0.185042(8)$.

To test the idea further, we have substituted in the infinite continued fraction

$$
f_{p_{\max }}(\chi) \mapsto \frac{1}{2}+\frac{1}{2} \sqrt{1+4 \gamma \chi}
$$

$p_{\max }$ varying from 9 to 27 . This amounts to taking $a_{p}$ constant, $a_{p}=\gamma \approx 0.1850$ for $p>p_{\max }$. Remarkably enough, for $\chi \rightarrow \infty$, the corresponding approximation for $E^{[\mathrm{qc}]}(\chi)$ behaves like $\chi^{3 / 2}$ with a coefficient close to the exact value $1 / 3$. We have thus adjusted $\gamma$ more precisely to get $1 / 3$ exactly. This yields a very stable sequence up to $p=25$, converging slowly toward the expected limit $0.18504 \ldots$.

$$
\begin{aligned}
& \gamma_{17}=0.1850687, \gamma_{18}=0.1850261, \gamma_{19}=0.1850603, \\
& \gamma_{20}=0.1850239, \gamma_{21}=0.1850562, \gamma_{22}=0.1850310 \\
& \gamma_{23}=0.1850562, \gamma_{24}=0.1850367, \gamma_{25}=0.185048(4) .
\end{aligned}
$$

corresponding to a singularity located at $\chi=\chi_{c} \approx$ -1.3504 , consistent with a direct analysis of the behaviour of the coefficients in the expansion (92). As a consequence, in all Tables and Figures we have reported results obtained from this approximated continued fraction.

\section{Spectrum for $\chi<0$}

The convergence of the various methods is rather poor for $\chi<0$, specially in the neighbourhood of the cut. Table VI displays a few results for $E^{[\mathrm{qc}]}(\chi)$. According to Table VI, the most precise results are in general obtained from the modified continued fraction, with a precision that for $|g|>1$ is comparable with the ODM method defined by Eq. (42a). Note that for the first value, which corresponds to $|g|=0.5$, the ODM method with $\alpha=5 / 4$ does not converge, as expected, and does not even provide a reliable estimate. The method of Sec. IVB is not useful either. Finally, in the neighbourhood of the singularity $\chi=\chi_{c}$ a calculation based on the determination of $\Delta_{01}$ and $S_{01}$ gives the most precise results (see Figs. 3 and 4).

The imaginary part after the cut for $\chi=-|\chi|+\mathrm{i} 0$ is necessarily negative when calculated from the approximated continued fraction. Therefore, the general consistency of the results coming from the continued fraction and the other ODM methods provides an additional confirmation of our conjecture about the sign of the discontinuity.

\section{CONCLUSIONS}

Let us briefly summarize the findings of the current numerical study of properties of the cubic anharmonic oscillator. For $g \geq 0$, the Hamiltonian (11) is $\mathcal{P} \mathcal{T}$ symmetric and has a real positive spectrum. The energy eigenvalues have divergent, Borel summable expansions in powers of the coupling constant $g$. The imaginary parts of the eigenvalues on the cut on the real negative axis are positive. Starting from the $\mathcal{P} \mathcal{T}$ symmetric case and developing a strong coupling expansion in the variable $\sqrt{\xi}=g^{-1 / 5}$, one sees that eigenvalues are univalued functions in some neighbourhood of the origin in the $\xi$ plane, 
TABLE VI: Values for $E^{[\mathrm{qc}]}(\chi)$, obtained by various ODM summation methods, on the real negative axis, at transformation order 150. Method (42a) and the continued fraction approximation (99) provide the best numerical convergence.

\begin{tabular}{cll}
\hline \hline \multicolumn{1}{c}{$\chi$} & \multicolumn{1}{c}{$-2^{4 / 5}=-1.741 \ldots$} & -1 \\
\hline Method of Eq. (42a) & \multicolumn{1}{c}{ no convergence } & $0.1957508157(1)$ \\
Method of Eq. (42b) & $0.42 \pm 0.20-(0.39 \pm 0.17) \mathrm{i}$ & $0.195(8)$ \\
Method of Eq. [42c) & $0.38(1)-0.36(8) \mathrm{i}$ & $0.195(5)$ \\
Continued fraction & $0.3898(5)-0.3644(3) \mathrm{i}$ & $0.1957508157161719(6)$ \\
\hline \hline$\chi$ & $-5^{4 / 5}=-0.275 \ldots$ & $-(21.6)^{-4 / 5}=-0.085 \ldots$ \\
\hline Method of Eq. (42a) & $0.282699258193274909899(0)$ & $0.34215801861934042140767(3)$ \\
Method of Eq. (42b) & $0.2826993(0)$ & $0.3421580(2)$ \\
Method of Eq. (42c) & $0.2827(1)$ & $0.34215(6)$ \\
Continued fraction & $0.2826992581932749098990(1)$ & $0.34215801861934042140767(6)$ \\
\hline \hline
\end{tabular}

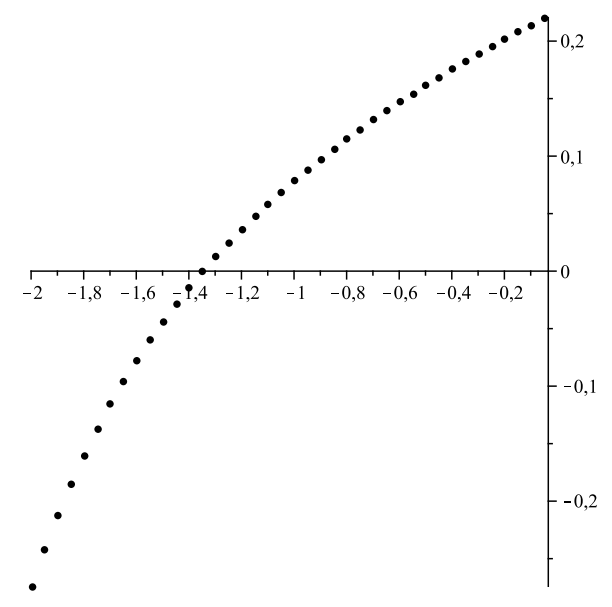

FIG. 3: $\Delta_{01}=\left(E_{\mathrm{GS}}^{[\mathrm{qc}]}-E_{\mathrm{ES}}^{[\mathrm{qc}]}\right)^{2} / 4$ as a function of $\chi$ for $-1.85 \leq \chi \leq-0.85$ [the value at the origin is $\Delta_{01}(\chi=0)=$ $0.2263073 \ldots]$.

with a simple pole at $z=\sqrt{\xi}=g^{-1 / 5}=0$ as a unique singularity.

In Sec. III, we have discussed various possible summation methods for the determination of energy eigenvalues of the cubic potential. Notably, we explore the numerical properties of three such methods here, given in Eqs. (42a), (42b) and (42c). The latter was used in our previous paper [4]. Summing the perturbative expansion of the ground state energy $E(g)$ by various implementations of the ODM method, we uncover additional properties. From the apparent convergence of the ODM method for $\alpha=5 / 2$ [see Eq. (42C)], we conclude that in the variable $z$ the function $E(g(z))$ has no other singularity in the sector $|\arg z|<\pi / 4$. In the variable $\chi=g^{-4 / 5}=z^{4}$, this translates into the property that the ground state

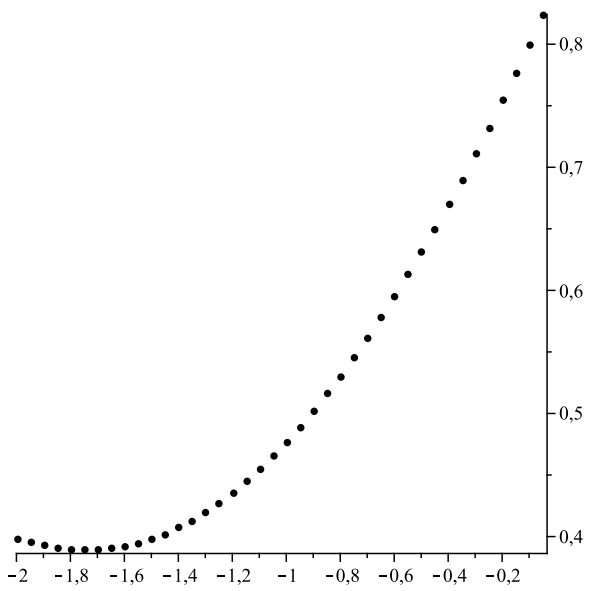

FIG. 4: $S_{01}=\left(E_{\mathrm{GS}}^{[\mathrm{qc}]}+E_{\mathrm{ES}}^{[\mathrm{qc}]}\right) / 2$ as a function of $\chi$ for $-1.85 \leq$ $\chi \leq-0.85$ [the value at the origin amount to $S_{01}(\chi=0)=$ 0.8482634 ...].

energy $E^{[\mathrm{qc}]}(\chi)$ of the Hamiltonian (5) also is a real analytic function in a cut plane with a cut along the real negative axis.

The ODM summation method (42a) converges very well in the strong coupling regime and thus allows us to determine a number of terms of the large $g$, thus small $\chi$ expansion. The precision can be further improved by the convergence acceleration algorithm (64) and, finally, by a combined calculation of the ground state and first excited energies. The first 20 terms are given in Eq. (92). A direct analysis of the strong coupling expansion indicates the existence of a square root singularity located at $\chi=$ $\chi_{c} \approx-1.351[g \approx 0.687 \exp (5 \mathrm{i} \pi / 4)$ in the second sheet $]$. These properties are confirmed by the direct calculation of the difference $\left(E_{\mathrm{GS}}-E_{\mathrm{ES}}\right)^{2}$, which vanishes linearly at 
the point $\chi_{c}=-1.3510415966(3)$, as given in Eq. (91).

The strong coupling expansion, in turn, can be summed by an expansion in a continued fraction (Sec. VIIB). The calculated coefficients of the continued fraction display an asymptotic behavior consistent with a square root singularity. The extrapolated continued fraction yields results in remarkable agreement with the more direct ODM calculations.

As a function of $z, E^{[\mathrm{qc}]}(\chi(z))$ is invariant under the rotation $z \mapsto z \exp (\mathrm{i} \pi / 2)$. It is thus entirely defined by its values for $-\pi / 4 \leq \arg (z) \leq+\pi / 4$, that is, $-5 \pi / 4 \leq$ $\arg (g) \leq+5 \pi / 4$. As a consequence, as a function of $\bar{z}$, $E$ has cuts only on the lines $\arg z=n \pi / 4$ where level merging can occur. Note that, from the point of view of Eq. (17), for $\chi<0$ the Hamiltonian is still $\mathcal{P} \mathcal{T}$ symmetric and, thus, the singularity at $\chi=\chi_{c}$ corresponds to a kind of spontaneous $\mathcal{P} \mathcal{T}$ symmetry breaking.

All coefficients of the associated expansion in a continued fraction are found to be positive as long as we can estimate them with enough precision, that is, up to order 27 (corresponding to a strong coupling expansion up to order 28), and seems to converge toward a positive value consistent with the square root singularity of the first level merging. This gives a very strong argument that the eigenvalue of the strong coupling Hamiltonian $\operatorname{Im} E^{[\mathrm{qc}]}(-|\chi|+\mathrm{i} 0)$ is negative on the cut. This conjecture is also consistent with the large $\chi$ behaviour, the Cauchy representation, the coefficient of the square root singularity. However, this property cannot be shared by all eigenvalues and may even be unique to the ground state.

To go beyond this study, one would have to apply these ODM summation methods more directly to the spectral equation, in order to eliminate all level merging singularities (for the pure cubic potential see Ref. [25]). Finally, we would like to emphasize the empirical evidence gathered during the current study, for the robustness of the ODM summation methods (42a), 42b) and (42c), which applied to different functions with varying implementation always give consistent results.

\section{Acknowledgements}

J.Z.-J. gratefully acknowledges CERN's hospitality, where a major part of this work was completed. U.D.J. acknowledges support by a Grant from the Missouri Research Board and by the National Science Foundation (Grant PHY-8555454).

\section{Appendix A: ODM and strong coupling expansion}

We assume that we know a perturbative expansion for $E(g)$ in powers of the coupling constant $g$, and that $E(g)$ also has a strong-coupling asymptotic expansion of the form,

$$
E(g)=g^{\beta} \sum_{n=0} \epsilon_{n} g^{-n / \alpha}, \quad g \rightarrow \infty,
$$

a property shared by the example (17) we discuss here. It is also shared by the quartic anharmonic oscillator [13] and all $x^{N}$ perturbations to the quantum harmonic oscillator. We then consider the conformal mapping (41),

$$
g=\rho \frac{\lambda}{(1-\lambda)^{\alpha}} .
$$

This transformation maps the real positive $g$ axis onto the finite $\lambda$ interval $[0,1]$. For $g \rightarrow \infty, \lambda \rightarrow 1$ and $g$ has an expansion at $\lambda=1$ of the form

$$
g^{-1 / \alpha}=\sum_{n=0} \Lambda_{n}(1-\lambda)^{n+1}
$$

with $\Lambda_{0}=\rho^{-1 / \alpha}$. The function

$$
\phi(\lambda)=(1-\lambda)^{\alpha \beta} E(g(\lambda))
$$

then has a Taylor series expansion at $\lambda=0$,

$$
\phi(\lambda)=\sum_{n=0} \phi_{n} \lambda^{n},
$$

as well as at $\lambda=1$,

$$
\phi(\lambda)=\sum_{n=0} \varphi_{n}(1-\lambda)^{n} .
$$

with $\varphi_{0}=\epsilon_{0} \rho^{\beta}$, where $\epsilon_{0}$ is the coefficient defined in (A1). This last property explains, to a large extent, the good convergence of the method even for $g \rightarrow \infty$.

\section{Appendix B: Large $g$ expansion: a few terms}

In order to determine the successive terms of the large $g$, small $\chi$ expansion we do not have to differentiate numerically the values of $E(g)$ calculated at $g$ large but finite. In the ODM method, the various terms have explicit analytic forms and we give here the first terms. First, we set

$$
\begin{aligned}
E(g) & =\phi(\lambda)(1-\lambda)^{-\alpha \beta} \\
& =\phi(\lambda)\left(\frac{g}{\rho}\right)^{\beta} \lambda^{-\beta} \equiv\left(\frac{g}{\rho}\right)^{\beta} \psi(\lambda) .
\end{aligned}
$$

Then we write the relation (A2) as

$$
\lambda=1-\left(\frac{\rho}{g}\right)^{1 / \alpha} \lambda^{1 / \alpha} .
$$


Setting $(\rho / g)^{1 / \alpha}=z$, we expand $s=1-\lambda$ in powers of $z$, so that $s \equiv 1-\lambda=z-\frac{1}{\alpha} z^{2}+\cdots$. The function $\psi(\lambda)$ can then be expanded successively in powers of $s$ and $z$ :

$$
\begin{aligned}
\psi(\lambda) & =\psi(1)-\psi^{\prime}(1) s+\frac{1}{2} \psi^{\prime \prime}(1) s^{2}+\cdots \\
& =\psi(1)-\psi^{\prime}(1) z+\left\{\frac{1}{2} \psi^{\prime \prime}(1)+\frac{\psi^{\prime}(1)}{\alpha}\right\} z^{2}+\cdots \\
& \left.=\rho^{\beta}\left(\epsilon_{0}+\rho^{-1 / \alpha} \epsilon_{1} z+\rho^{-2 / \alpha} \epsilon_{2} z^{2}+\cdots\right), \quad \text { (B3 }\right)
\end{aligned}
$$

where the $\epsilon_{n}$ coefficients are given in Eq. A1). The first terms yield

$$
\begin{aligned}
& \epsilon_{0}=\psi(1) \rho^{-\beta}, \\
& \epsilon_{1}=-\psi^{\prime}(1) \rho^{-\beta+1 / \alpha}, \\
& \epsilon_{2}=\left\{\frac{1}{2} \psi^{\prime \prime}(1)+\frac{\psi^{\prime}(1)}{\alpha}\right\} \rho^{-\beta+2 / \alpha}, \\
& \epsilon_{3}=-\left\{\frac{3-\alpha}{2 \alpha^{2}} \psi^{\prime}(1)+\frac{\psi^{\prime \prime}(1)}{\alpha}+\frac{\psi^{\prime \prime \prime}(1)}{6}\right\} \rho^{-\beta+3 / \alpha} .
\end{aligned}
$$

\section{Appendix C: Numerical evidence for strong coupling}

In Ref. 22], we started from the Hamiltonian

$$
H=-\frac{1}{2} \frac{\partial^{2}}{\partial x^{2}}+\frac{1}{2} x^{2}+\sqrt{g} x^{3} .
$$

which entails both a change in the normalization of the coupling term and also a change in the complex phase of the coupling. The transformation $x \rightarrow g^{-1 / 10} x$ brings the Hamiltonian into the form

$$
H=g^{1 / 5}\left(-\frac{1}{2} \frac{\partial^{2}}{\partial x^{2}}+x^{3}+\frac{x^{2}}{2 g^{5 / 2}}\right) .
$$

We now define $g=u^{-5 / 2}$ and write

$$
H=g^{1 / 5}\left(-\frac{1}{2} \frac{\partial^{2}}{\partial x^{2}}+x^{3}+u \frac{x^{2}}{2}\right) .
$$

A shift of the variable $x \rightarrow x-\frac{1}{6} u$ then results in

$$
\begin{aligned}
H & =g^{1 / 5}\left(-\frac{1}{2} \frac{\partial^{2}}{\partial x^{2}}+x^{3}-\frac{u^{2}}{12} x+\frac{u^{3}}{180}\right) \\
& =g^{1 / 5}\left(-\frac{1}{2} \frac{\partial^{2}}{\partial x^{2}}+x^{3}-\frac{1}{12} g^{-4 / 5} x+\frac{1}{180} g^{-6 / 5}\right)
\end{aligned}
$$

If one now writes the strong-coupling expansion of the $N$ th energy level in the form of Eq. (17) of Ref. 22],

$$
E_{N}(g)=g^{1 / 5} \sum_{K=0}^{\infty} L_{N, K} g^{-2 K / 5},
$$

then the terms [see Eq. CC4 ]

$$
-\frac{1}{2} \frac{\partial^{2}}{\partial x^{2}}+x^{3}-\frac{1}{12} g^{-4 / 5} x
$$

generate a convergent series in even powers of $g^{-4 / 5}$, and the term

$$
\frac{1}{180} g^{-6 / 5}
$$

in Eq. (C4) is the only term of odd power in $g^{-2 / 5}$ in Eq. (C5). From the point of view of the strong coupling expansion, this term $L_{N, 3}=1 / 108$ represents a shift, independent of the quantum number $N$, of all levels of the cubic potential. This affords an explanation for the observations made in Table 2 of Ref. [22], where on the basis of numerical evidence, it was conjectured that the terms of order $K=1$ according Eq. (C5) vanish, and that the numerical value for the coefficient of the term of order $K=3$ is $1 / 108$ uniformly for all levels.
[1] C. M. Bender and S. Boettcher, Real spectra in nonHermitian Hamiltonian having $\mathcal{P} \mathcal{T}$ symmetry, Phys. Rev. Lett. 80 (1998) 5243-5246.

[2] P. Dorey, C. Dunning and R. Tateo, Spectral equivalences, Bethe ansatz equations, and reality properties in $\mathcal{P} \mathcal{T}$-symmetric quantum mechanics, J. Phys. A 34 L391 (2001); ibid. 34 (2001) 5679-5704.

[3] L. C. Shin, On the reality of eigenvalues for a class of $\mathcal{P} \mathcal{T}$-symmetric oscillators, Commun. Math. Phys. 229 (2002) 543-564.

[4] J. Zinn-Justin and U. D. Jentschura, Order-dependent mappings: strong coupling behaviour from weak coupling expansions in non-Hermitian theories, J. Math. Phys. 51 (2010) 072106.

[5] E. Caliceti, S. Graffi, and M. Maioli, Perturbation theory of odd anharmonic oscillators, Commun. Math. Phys. 75 (1980) 51-66.

[6] E. Caliceti, Distributional Borel summability of odd anharmonic oscillators, J. Phys. A 33 (2000) 3753-3770.

[7] L. N. Lipatov, Divergence of the perturbation-theory series and pseudoparticles, JETP Lett. 25 (1977) 104107; Divergence of the perturbation-theory series and the 
quasi-classical theory, Sov. Phys. JETP 45 (1977) 216223 .

[8] E. Brézin, J. C. Le Guillou, J. Zinn-Justin, Perturbation theory at large order. I. The $\varphi^{N}$ interaction, Phys. Rev. D 15 (1977) 1544-1557.

[9] J. Zinn-Justin, Perturbation series at large orders in quantum mechanics and field theories: application to the problem of resummation, Phys. Rep. 70 (1981) 109-167.

[10] U. D. Jentschura, A. Surzhykov, J. Zinn-Justin, Generalized nonanalytic expansions, $\mathcal{P} \mathcal{T}$-symmetry and large order formulas for odd anharmonic oscillators, SIGMA 5 (2009) 005.

[11] V. Grecchi, M. Maioli and A. Martinez, Padé summability of the cubic oscillator, J. Phys. A 42 (2009) 425208.

[12] C. M. Bender, E. J. Weniger, Numerical evidence that the perturbation expansion for a non-Hermitian $\mathcal{P} \mathcal{T}$ symmetric Hamiltonian is Stieltjes, J. Math. Phys. 42 (2001) 2167-2183.

[13] R. Seznec, J. Zinn-Justin, Summation of divergent series by order dependent mappings: Application to the anharmonic oscillator and critical exponents in field theory, J. Math. Phys. 20 (1979) 1398-1408.

[14] J. Zinn-Justin, Summation of divergent series: Orderdependent mapping, arXiv:1001.0675 [math-ph].

[15] F. M. Fernandez, R. Guardiola, J. Ros and M. Znojil, Strong-coupling expansions for the $\mathcal{P} \mathcal{T}$-symmetric oscillators $V(x)=a(\mathrm{i} x)+b(\mathrm{i} x)^{2}+c(\mathrm{i} x)^{3}$, J. Phys. A: Math. Gen. 31 (1998) 10105-10112.
[16] U. D. Jentschura, J. Zinn-Justin, Calculation of the Characteristic Functions of Anharmonic Oscillators, arXiv:1001.4313 [math-ph].

[17] C.M. Bender and T.T. Wu, Anharmonic oscillator, Phys. Rev. 184 (1969) 1231-1260.

[18] E. Delabaere and D. T. Trinh, Spectral analysis of the complex cubic oscillator, J. Phys. A 33 (2000) 8771-8796.

[19] H. Kleinert, W. Janke, Convergence behavior of variational perturbation expansion: A method for locating Bender-Wu singularities, Phys. Lett. A 206 (1995) 283289.

[20] M. E. Fisher, Yang-Lee Edge Singularity and $\phi^{3}$ Field Theory, Phys. Rev. Lett. 40 (1978) 1610-1613.

[21] C. M. Bender, D. C. Brody and H.F. Jones, Scalar Quantum Field Theory with Cubic Interaction, arXiv: hep-th/0402011

[22] U. D. Jentschura, A. Surzhykov, M. Lubasch, and J. Zinn-Justin, Structure, Time Propagation and Dissipative Terms for Resonances, J. Phys. A 41 (2008) 095302.

[23] D. Masoero, Poles of Intégrale Tritronquéée and Anharmonic Oscillators. A WKB Approach, arXiv:0909.5537v2 [math.CA].

[24] R. Guida, L. Konishi, H. Suzuki, Improved Convergence Proof of the Delta Expansion and Order Dependent Mappings, Ann. Phys. (N.Y.) 249 (1996) 109-145.

[25] A. Voros, Airy function - exact WKB results for potentials of odd degree, J. Phys. A 32 (1999) 1301-1311. 